\title{
Adjunct zonisamide to levodopa for DLB parkinsonism
}

\author{
A randomized double-blind phase 2 study
}

Miho Murata, MD, PhD, Toshinari Odawara, MD, PhD, Kazuko Hasegawa, MD, PhD, Sayaka liyama, Masatoshi Nakamura, Masaaki Tagawa, PhD, and Kenji Kosaka, MD, PhD

Neurology ${ }^{\circledR}$ 2018;90:e664-e672. doi:10.1212/WNL.0000000000005010

\section{Abstract}

\section{Objective}

To investigate the efficacy and safety of zonisamide as an adjunct to levodopa therapy for parkinsonism in patients with dementia with Lewy bodies (DLB).

\section{Methods}

This phase 2, placebo-controlled, randomized, double-blind study consisted of run-in (placebo, 4 weeks) and treatment (placebo or zonisamide 25 or $50 \mathrm{mg}$ once daily, 12 weeks) periods. Outpatients diagnosed with probable DLB were eligible for inclusion. The primary endpoint was the change from baseline in Unified Parkinson's Disease Rating Scale (UPDRS) part 3 total score at week 12. Cognitive function, behavioral and psychological symptoms of dementia (BPSD), caregiver burden, other UPDRS parts as secondary endpoints, and safety were also assessed.

\section{Results}

Overall, 158 patients with DLB received the study drug; 21 discontinued during treatment and 137 completed treatment. Improvement in UPDRS part 3 total score at week 12 was significantly greater in the zonisamide $50 \mathrm{mg}$ group compared with placebo (between-group difference $-4.1 ; 95 \%$ confidence interval -6.8 to $-1.4 ; p=0.003)$. Zonisamide did not worsen cognitive function, BPSD, or caregiver burden. The overall incidence of adverse events was higher in the zonisamide $50 \mathrm{mg}$ than the $25 \mathrm{mg}$ and placebo groups (65.3\%, 43.1\%, and 50.0\%, respectively); similar rates of serious adverse events were observed among all groups.

\section{Conclusion}

Zonisamide (adjunctive to levodopa) improved parkinsonism accompanying DLB without worsening cognitive function or psychiatric symptoms.

\section{Clinical trial registration}

JapicCTI-122040.

\section{Classification of evidence}

This study provides Class I evidence that zonisamide (adjunctive to levodopa) improves parkinsonism and is well-tolerated in patients with DLB.

\section{Correspondence \\ Dr. Murata \\ mihom@ncnp.go.jp}

\section{MORE ONLINE}

$\rightarrow$ Class of Evidence

Criteria for rating

therapeutic and diagnostic

Studies

NPub.org/coe

\section{- CME Course}

NPub.org/cmelist

\section{RELATED ARTICLE}

\section{Editorial}

Zonisamide for DLB parkinsonism: An old drug used in a new context

Page 349 


\section{Glossary}

$\mathbf{A E}=$ adverse event; $\mathbf{A N C O V A}=$ analysis of covariance $\mathbf{B P S D}=$ behavioral and psychological symptoms of dementia; $\mathbf{C I}=$ confidence interval; DCI = decarboxylase inhibitor; DLB = dementia with Lewy bodies; LOCF = last observation carried forward; LSM = least squares mean; $\mathbf{m I T T}=$ modified intention-to-treat; $\mathbf{M M R M}=$ mixed-effect model repeated measures; MMSE = Mini-Mental State Examination; NPI-10 = Neuropsychiatric Inventory-10; PD $=$ Parkinson disease; PDD = Parkinson disease with dementia; PP = per protocol; UPDRS = Unified Parkinson's Disease Rating Scale; ZBI = Zarit Burden Interview.

Dementia with Lewy bodies (DLB) is thought to be the second most common type of dementia after Alzheimer disease, accounting for $10 \%-15 \%$ of all patients with dementia. ${ }^{1}$ Patients with DLB frequently complain of common symptoms including hallucinations and parkinsonism, as well as other symptoms such as REM sleep behavior disorder, severe neuroleptic sensitivity, and autonomic nervous system disorders. ${ }^{1}$ DLB is one phenotype of Lewy body disease, which includes Parkinson disease (PD), and is characterized by the expression of Lewy bodies in the central and autonomic nervous systems. Furthermore, treatment with levodopa is beneficial for symptoms of parkinsonism accompanying DLB. However, conventional anti-Parkinson drugs including levodopa should be prescribed with care because they can induce psychiatric symptoms, such as hallucinations and delusions. $^{2}$

Zonisamide (1,2-benzisoxazole-3-methanesulfonamide), widely used for epilepsy, has been available as an antiParkinson drug (adjunctive agent in levodopa treatment) in Japan since 2009. In 4 placebo-controlled, randomized trials, ${ }^{3-6}$ zonisamide improved motor symptoms and the "wearing-off" phenomenon, with a low incidence of motor complications such as dyskinesia or psychiatric symptoms such as hallucinations. The mechanism of action of zonisamide on PD has not been fully elucidated, but several studies reported that zonisamide has multiple functions in the dopaminergic (activation of dopamine synthesis and release $^{7}$ and inhibition of monoamine oxidase $\mathrm{B}^{8}$ ) and nondopaminergic (blockade of sodium channels, ${ }^{9}$ T-type calcium channels, ${ }^{10,11}$ and GABAergic transmission via striatal opioid $\delta 1$-receptor-associated interactions ${ }^{12}$ ) pathways.

A previous study of 3 patients with DLB reported that zonisamide improved parkinsonism and reduced caregiver burden without deteriorating cognitive function or behavioral or psychological symptoms ${ }^{13}$; these results were supported in 2 case studies of 1 patient each. ${ }^{14,15}$ Therefore, we hypothesized that zonisamide would be efficacious for parkinsonism accompanying DLB without psychiatric deterioration.

We conducted a phase 2, placebo-controlled, randomized, double-blind study to investigate the efficacy and safety of zonisamide in patients with DLB.

\section{Methods}

\section{Participants}

\section{Inclusion and exclusion criteria at the start of the run- in period}

Outpatients diagnosed with probable DLB based on the 2005 version of the clinical diagnostic criteria for $\mathrm{DLB}^{2}$ who satisfied all of the following inclusion criteria and who did not meet any of the exclusion criteria were eligible for this study.

Major inclusion criteria included age 20-84 years; Unified Parkinson's Disease Rating Scale (UPDRS) ${ }^{16}$ part 3 total score $\geq 10$; Mini-Mental State Examination $(\mathrm{MMSE})^{17}$ total score 10-26; and administration of levodopa/decarboxylase inhibitor (DCI) for $\geq 12$ weeks before the run-in period with the treatment regimen unchanged for the last 2 weeks.

Patients using antidementia drugs with no changes in the dose/type of drugs administered for $\geq 12$ weeks before the run-in period were eligible. Patients using anti-Parkinson drugs other than levodopa/DCI, antihypertensive drugs, other CNS drugs, cardiovascular system drugs, gastrointestinal system drugs, or Yokukansan (a traditional Japanese herbal medicine) were eligible, providing there were no changes in the dose/type of these drugs administered for $\geq 2$ weeks before the run-in period.

Based on the DLB diagnostic criteria, ${ }^{2}$ we defined PD with dementia (PDD) as well-established PD (i.e., as diagnosed by a physician) followed by onset of dementia. Patients with PDD or Parkinson syndromes other than DLB, patients who did not respond to levodopa therapy, patients with a history of treatment with zonisamide, and patients with epilepsy were excluded from the study.

\section{Inclusion and exclusion criteria at the start of the treatment period}

Patients remained eligible if they had a UPDRS part 3 total score $\geq 10$ with no changes in the dose/type of restricted coadministered drugs such as anti-Parkinson drugs, antihypertensive drugs, other CNS drugs, cardiovascular system drugs, gastrointestinal system drugs, or Yokukansan for the run-in period. Patients for whom the UPDRS part 3 total score changed by $\geq 20$ from the initiation of the run-in period and for whom the medicated rate was $<80 \%$ were excluded. 


\section{Trial design and treatments}

This multicenter, placebo-controlled, randomized, doubleblind, parallel-group comparison study compared the efficacy of zonisamide ( 25 and $50 \mathrm{mg}$ once daily) with placebo for treating parkinsonism in patients with DLB. The study consisted of a run-in period (4 weeks) and a treatment period (12 weeks). Placebo tablets were orally administered once daily for 4 weeks during the run-in period under single-blind conditions. The objective of the run-in period was to eliminate any potential confounding effects of other anti-Parkinson drugs.

The eligibility of patients was confirmed immediately before the treatment period (baseline, week 0 ). Eligible patients were randomly allocated to 1 of 3 groups, and administered placebo or zonisamide 25 or $50 \mathrm{mg}$ once daily under double-blind conditions. Participants visited the study sites every 4 weeks, for a total of 5 visits.

The dosage and administration were unchanged throughout the treatment period for test drugs, from 12 weeks before the run-in period throughout the treatment period for antidementia drugs, and from 2 weeks before the run-in period throughout the treatment period for levodopa/DCI, other anti-Parkinson drugs, and drugs listed in the Participants section. Starting new drugs of these classes during the study was prohibited. For anti-Parkinson drugs, dose reduction was allowed when motor complications such as dyskinesia and nonmotor adverse events (AEs) occurred, but dose reescalation was prohibited. Cessation or discontinuation of levodopa/DCI and coadministration of zonisamide, benzamide antipsychotics, or other investigational new drugs were prohibited.

This study was conducted at 60 medical institutions between March 2013 and April 2014.

\section{Standard protocol approvals, registration, and patient consent}

This study was reviewed and approved by the institutional review board of each study site. It was conducted in compliance with the Declaration of Helsinki, clinical study protocol, Good Clinical Practice, and applicable regulations. All patients/proxy consenters and caregivers provided written informed consent. This study was registered with the Japan Pharmaceutical Information Center (JapicCTI-122040).

\section{Randomization and blinding}

The study drug allocation table was prepared by the person responsible for study drug allocation, securely sealed, and stored until study completion. Patients were allocated using a verified program (SAS 9.1; SAS Institute Inc., Cary, NC) with 3 patients allocated to each block (1 patient per group) to ensure a ratio of 1:1:1 for placebo and zonisamide 25 and $50 \mathrm{mg}$ groups. Block size was not disclosed to investigators. Concealment of allocation sequence and blindness (for all patients, caregivers, and attending physicians) were ensured by double-dummy method and indistinguishability of study drugs was confirmed before study initiation and at code breaking. Randomization key codes were appropriately stored until database lock.

\section{Outcomes}

For efficacy analyses, the primary endpoint was change from baseline in UPDRS part 3 total score at week 12. Secondary endpoints were changes from baseline in total scores of UPDRS and each UPDRS part (parts 1, 2 ["on"], 4, and 1-4), and scores of each UPDRS item at each evaluation time point. Changes from baseline in total scores of the MMSE, Neuropsychiatric Inventory-10 (NPI-10), ${ }^{18}$ and Zarit Burden Interview (ZBI), ${ }^{19}$ as the respective evaluation indices of cognitive function, behavioral and psychological symptoms of dementia (BPSD), and caregiver burden were also evaluated as secondary endpoints at week 12 .

UPDRS was evaluated every 4 weeks ( 5 times in total). MMSE, NPI-10, and ZBI were evaluated at the start of the run-in period and at the beginning/end of the treatment period ( 3 times in total). The baseline was defined as the start of the treatment period for the primary endpoint; for secondary endpoints, if the data were missing at that time, the baseline was defined as the start of the run-in period. The sponsor provided training to investigators for use of the UPDRS.

For safety analyses, laboratory test values, vital signs, and body weight were measured at each visit, and 12-lead ECG at rest was measured at screening, baseline, and weeks 4 and 12. AEs observed between initiation and the end of the treatment period were evaluated by each investigator, who recorded the following information: type of $\mathrm{AE}$, date of onset, seriousness/severity, change (if any) made to the study drug in response to the $\mathrm{AE}$, treatment of the $\mathrm{AE}$, outcome, and causal relationship to the study drug. The investigator followed up with the patient until the $\mathrm{AE}$ resolved or improved. If serious AEs occurred, the investigator immediately alerted the director at the investigator's institution in writing and the study sponsor. The schedule is shown in table e-1 (links.lww.com/WNL/A180) and the study flow is illustrated in figure e-1 (links.lww.com/WNL/A181).

\section{Statistical analysis}

The primary analysis population consisted of patients receiving at least one dose of study drug for the treatment period with UPDRS part 3 total scores at baseline and any score after the start of the treatment period (modified intention-to-treat [mITT] population).

Superiority of zonisamide ( 25 and $50 \mathrm{mg}$ ) over placebo was verified by analysis of covariance (ANCOVA) with treatment groups as fixed effects and baseline values as covariates. Missing values for each UPDRS score at week 12 were supplemented using the last observation carried forward (LOCF) approach.

Change from baseline was calculated as least squares mean $(\mathrm{LSM}) \pm$ SEM. 
Between-group differences (vs placebo) were presented as LSM with 95\% confidence intervals (CIs) and $p$ values. For the primary endpoint, multiplicity of comparisons of the zonisamide groups and placebo was adjusted using the Fisher least significant difference method.

ANCOVA was applied to the per protocol (PP) population, and mixed-effect model repeated measures (MMRM) with treatment groups and visits as fixed effects, baseline values as covariates, and interaction between the groups and visits was applied to the mITT population for sensitivity analyses. The sample size calculation is described in the supplementary information (links.lww.com/WNL/A182).

\section{Results}

\section{Patients}

Although the study inclusion criterion was age $20-84$ years, the actual age range of patients enrolled was $56-84$ years. Of 173 patients screened, 15 discontinued during the run-in period, and 158 were randomized to receive the allocated study drug (figure 1). Twenty-one patients discontinued during the treatment period and 137 completed treatment.

The discontinuation rate during the treatment period was moderately higher in the zonisamide groups ( 9 patients [17.6\%] for $25 \mathrm{mg}$ and 7 [14.3\%] for $50 \mathrm{mg}$ ) compared with placebo $(5[8.6 \%])$.
The mITT population consisted of 152 patients (55, 48, and 49 in placebo and zonisamide 25 and $50 \mathrm{mg}$ groups, respectively) after excluding 6 patients who lacked a UPDRS part 3 total score at baseline or in the treatment period. All 158 randomized patients $(58,51$, and 49 in placebo and zonisamide 25 and $50 \mathrm{mg}$ groups, respectively) were included in the safety analysis.

In the mITT population, approximately $60 \%$ were men, and the mean age was 75.1 years. Those aged $<65$ years and $\geq 75$ years constituted $<10 \%$ and $>60 \%$ of patients, respectively. The mean duration after diagnosis of DLB was 1.5 years and the mean durations of motor symptoms and dementia were 3.6 and 3.8 years, respectively. Of the core symptoms defined in the 2005 version of clinical diagnostic criteria for $\mathrm{DLB},{ }^{2}$ fluctuating cognition and visual hallucinations were each present in approximately $70 \%$ of patients and motor dysfunction was present in all patients. Levodopa dose and levodopa equivalent daily dose were $279 \pm 149$ and $319 \pm 192 \mathrm{mg} / \mathrm{d}$, respectively. Dopamine agonists were used by $19.7 \%$ of patients and other anti-Parkinson drugs by $<10 \%$ per drug. Antidementia and CNS drugs were used in $75.0 \%$ and $37.5 \%$ of patients, respectively.

Baseline disease-related variables were similar among the 3 groups, except for a slightly higher proportion of patients aged $\geq 75$ years in the placebo group and a slightly lower mean NPI10 total score at baseline in the zonisamide $25 \mathrm{mg}$ group (table 1).

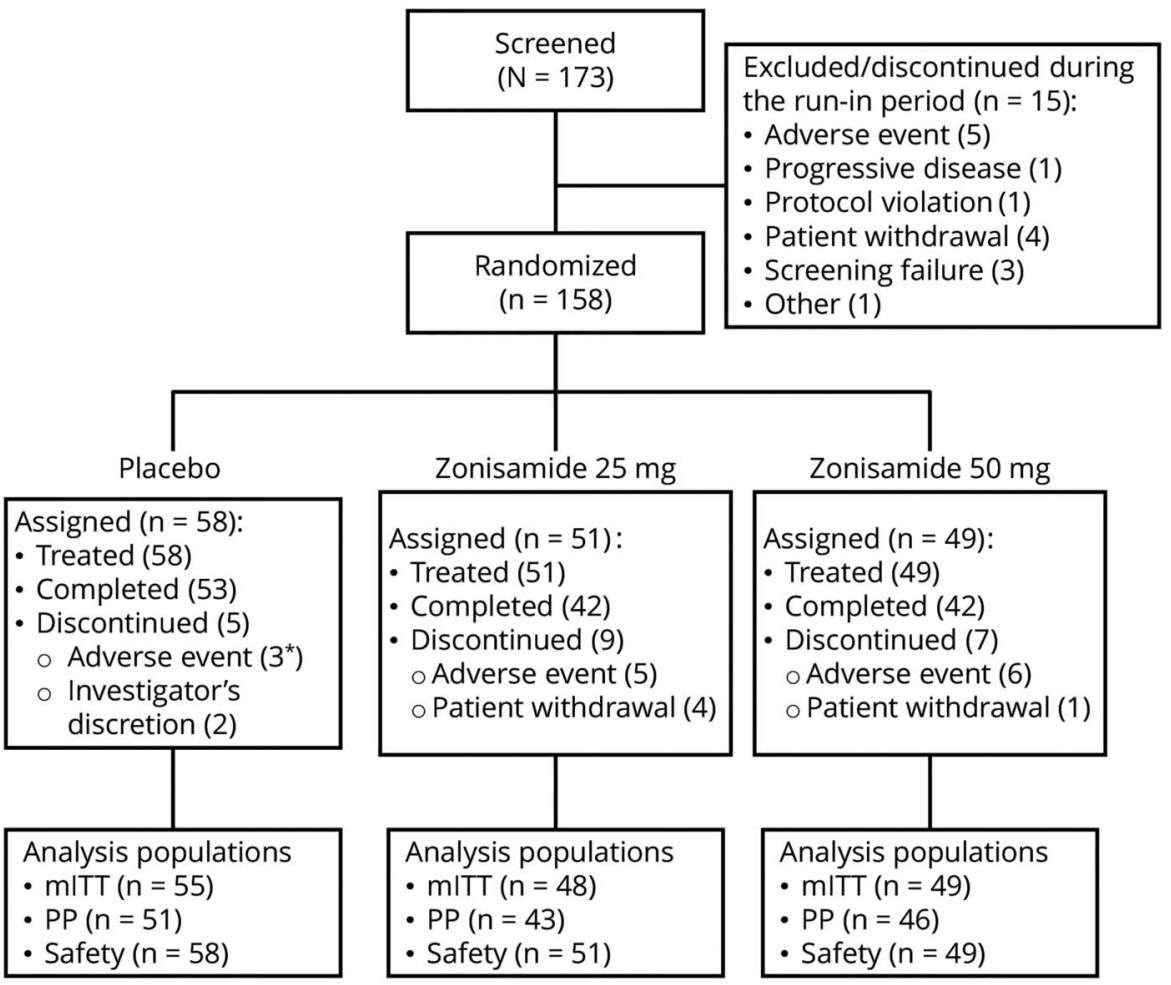

*An adverse event occurred in the run-in period in 1 of 3 patients (excluded from table 2). mITT = modified intention-to-treat; PP = per protocol. 


\section{Efficacy}

UPDRS part 3 total scores were decreased at week 12 (LOCF) in all groups compared with baseline, indicating improved parkinsonism. Changes from baseline (LSM \pm SEM) were $-2.1 \pm 0.9,-4.4 \pm 1.0$, and $-6.2 \pm 1.0$ in placebo and zonisamide 25 and $50 \mathrm{mg}$ groups, respectively; the change in UPDRS part 3 total score was significantly greater in the zonisamide $50 \mathrm{mg}$ group (between-group difference -4.1; $95 \% \mathrm{CI}-6.8$ to $-1.4 ; p=0.003)$ and greater in the zonisamide $25 \mathrm{mg}$ group (between-group difference -2.3 ; $95 \% \mathrm{CI}-5.0$ to $0.4 ; p=0.099$ ) compared with placebo (figure 2 and table e-2, links.lww.com/WNL/A180).

Significant changes in UPDRS part 3 total scores were observed between zonisamide $50 \mathrm{mg}$ and placebo by ANCOVA of the PP population (between-group difference -4.4 ; 95\% CI -7.2 to $-1.5 ; p=0.003)$ and MMRM analysis of the mITT population (between-group difference $-4.7 ; 95 \% \mathrm{CI}-7.5$ to $-1.9 ; p=0.001)$.

The change in UPDRS part 3 total scores over time is shown in figure 3. Although a decrease in the UPDRS part 3 total score was observed from baseline at week 4 in all groups, the score remained constant up to week 12 for placebo in contrast with further decreases in the zonisamide groups. In the zonisamide $50 \mathrm{mg}$ group, the score was significantly lower at week 8 (between-group difference $-2.8 ; 95 \% \mathrm{CI}-5.3$ to $-0.4 ; p=0.022$ ) and week 12 (between-group difference -5.1 ; $95 \%$ CI -8.0 to $-2.2 ; p<0.001)$ compared with placebo. The change from baseline for each total score for UPDRS part 1, 2 ("on"), 4, or 1-4 at week 12 (LOCF) in the zonisamide groups was similar to placebo (table e-2, links.lww.com/ WNL/A180). The total scores for MMSE, NPI-10, and ZBI did not change significantly from baseline in any group

Figure 2 Change from baseline in Unified Parkinson's Disease Rating Scale (UPDRS) part 3 total score at week 12 (last observation carried forward [LOCF])

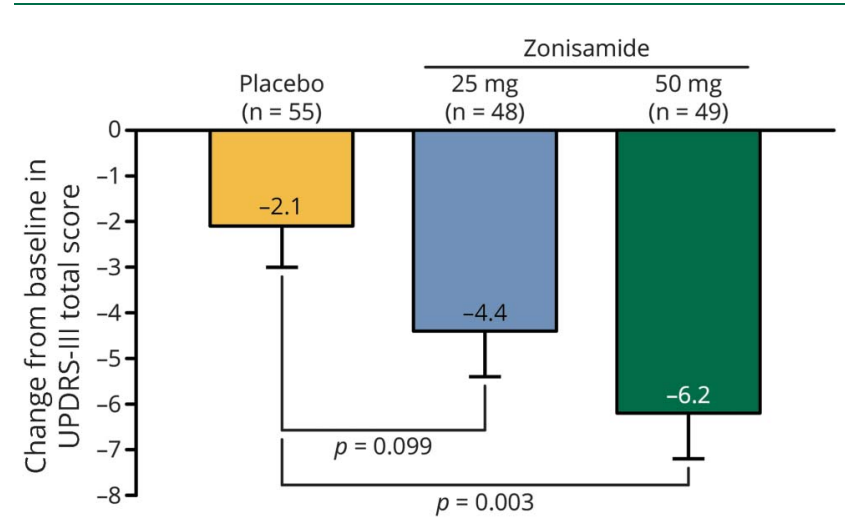

Results are presented as least squares mean \pm SEM with LOCF. Statistical analysis was performed by analysis of covariance with Fisher least significant difference method for multiplicity adjustment $(p<0.05$ : statistically significant vs placebo).
Figure 3 Time course of change from baseline in Unified Parkinson's Disease Rating Scale (UPDRS) part 3 total score

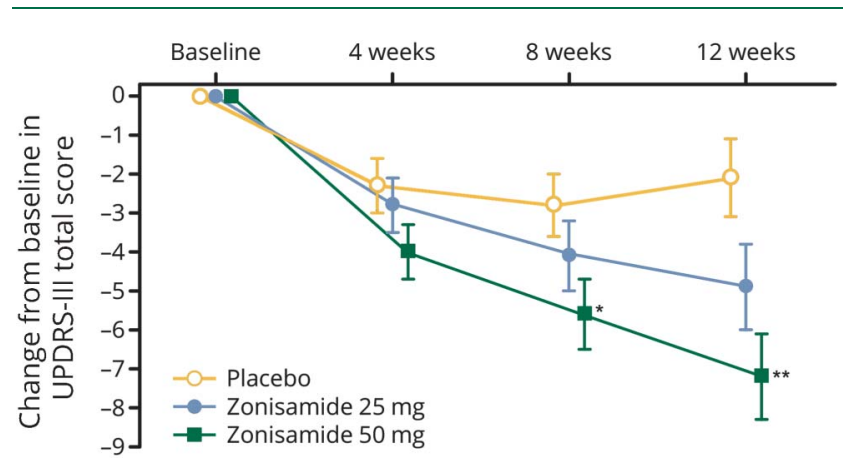

Results are the least squares mean \pm SEM. Statistical analysis at weeks 4, 8, and 12 was performed by analysis of covariance. ${ }^{*} p<0.05$; ${ }^{*} p<0.001$ (vs placebo).

(table e-2), suggesting administration of zonisamide did not worsen cognitive function, BPSD, or caregiver burden.

Scores at baseline and changes from baseline at week 12 for each item of UPDRS part 3, MMSE, and NPI-10 are shown in tables e-3, e-4, and e-5 (links.lww.com/WNL/A180), respectively.

\section{Safety}

The overall incidence of AEs was comparable between zonisamide $25 \mathrm{mg}$ and placebo but was higher for zonisamide $50 \mathrm{mg}$ ( $50.0 \%, 43.1 \%$, and $65.3 \%$ for placebo and zonisamide 25 and $50 \mathrm{mg}$, respectively) (table 2). The number of discontinuations because of AEs was higher with zonisamide 25 and $50 \mathrm{mg}$ than with placebo and included 2 patients ( 2 events) receiving placebo, 5 patients (6 events) receiving zonisamide $25 \mathrm{mg}$, and 6 patients (6 events) receiving zonisamide $50 \mathrm{mg}$. The AEs leading to discontinuations were colonic volvulus and anxiety disorder in the placebo group; somnolence, upper abdominal pain, dysgeusia, drug eruption, lung adenocarcinoma, and pneumonia in the zonisamide $25 \mathrm{mg}$ group; and back pain, hallucinations, ileus, cognitive disorder, rash, and decreased appetite in the zonisamide $50 \mathrm{mg}$ group.

Serious AEs occurred in 3 patients ( 3 events), 4 patients ( 5 events), and 3 patients ( 4 events) in the placebo and zonisamide 25 and $50 \mathrm{mg}$ groups, respectively. The serious AEs were dysphagia, aspiration pneumonia, lung adenocarcinoma, pneumonia, and rectal cancer in the zonisamide $25 \mathrm{mg}$ group, and back pain, ileus, decreased appetite, and chondrocalcinosis pyrophosphate (suspected pseudogout) in the zonisamide $50 \mathrm{mg}$ group. A causal relationship with the study drug was denied for all events except decreased appetite.

The incidences of common drug-related AEs (weight decreased, appetite decreased, and rash) were higher with 
Table 1 Patient characteristics (modified intention-to-treat population)

\begin{tabular}{|c|c|c|c|c|}
\hline & \multirow[b]{2}{*}{ Placebo $(n=55)$} & \multicolumn{2}{|c|}{ Zonisamide } & \multirow[b]{2}{*}{ Total $(n=152)$} \\
\hline & & $25 \mathrm{mg}(\mathrm{n}=48)$ & $50 \mathrm{mg}(\mathrm{n}=49)$ & \\
\hline \multicolumn{5}{|l|}{ Baseline characteristics } \\
\hline Male sex & $31(56.4)$ & $30(62.5)$ & $28(57.1)$ & 89 (58.6) \\
\hline \multicolumn{5}{|l|}{ Age, y } \\
\hline Mean \pm SD & $76.3 \pm 6.8$ & $74.3 \pm 5.5$ & $74.6 \pm 6.6$ & $75.1 \pm 6.4$ \\
\hline Range & $56-84$ & $62-84$ & $59-84$ & $56-84$ \\
\hline$\geq 65$ & $52(94.5)$ & $44(91.7)$ & $44(89.8)$ & $140(92.1)$ \\
\hline$\geq 75$ & $40(72.7)$ & $25(52.1)$ & $29(59.2)$ & $94(61.8)$ \\
\hline DLB duration, y & $1.5 \pm 1.6$ & $1.6 \pm 1.9$ & $1.5 \pm 1.7(n=48)$ & $1.5 \pm 1.7(n=151)$ \\
\hline Duration of motor symptoms, y & $3.8 \pm 3.3(n=53)$ & $3.5 \pm 3.0(n=47)$ & $3.5 \pm 2.5$ & $3.6 \pm 2.9(n=149)$ \\
\hline Dementia duration, y & $3.9 \pm 2.5(n=54)$ & $3.7 \pm 2.4(n=46)$ & $3.7 \pm 2.5(n=47)$ & $3.8 \pm 2.5(n=147)$ \\
\hline \multicolumn{5}{|l|}{ Diagnostic criteria for DLB } \\
\hline \multicolumn{5}{|l|}{ Core features } \\
\hline Fluctuating cognition & $35(63.6)$ & $33(68.8)$ & $34(69.4)$ & $102(67.1)$ \\
\hline Visual hallucinations & $38(69.1)$ & $33(68.8)$ & $32(65.3)$ & $103(67.8)$ \\
\hline Parkinsonism & $55(100)$ & $48(100)$ & $49(100)$ & $152(100)$ \\
\hline \multicolumn{5}{|l|}{ Suggestive clinical features } \\
\hline REM sleep behavior disorder & $31(56.4)$ & $20(41.7)$ & $21(42.9)$ & $72(47.4)$ \\
\hline Severe neuroleptic sensitivity & $8(14.5)$ & $9(18.8)$ & $10(20.4)$ & $27(17.8)$ \\
\hline \multicolumn{5}{|l|}{ Concomitant drugs } \\
\hline Levodopa dose, mg/d & $267 \pm 155$ & $279 \pm 151$ & $292 \pm 142$ & $279 \pm 149$ \\
\hline LEDD, $\mathrm{mg} / \mathrm{d}$ & $299 \pm 182$ & $334 \pm 228$ & $326 \pm 164$ & $319 \pm 192$ \\
\hline MAO-B inhibitor & $2(3.6)$ & $5(10.4)$ & $4(8.2)$ & $11(7.2)$ \\
\hline Amantadine & $1(1.8)$ & $3(6.3)$ & $5(10.2)$ & $9(5.9)$ \\
\hline Dopamine agonist & $12(21.8)$ & $9(18.8)$ & $9(18.4)$ & $30(19.7)$ \\
\hline A2A antagonist & $0(0.0)$ & $0(0.0)$ & $0(0.0)$ & $0(0.0)$ \\
\hline Droxidopa & $1(1.8)$ & $1(2.1)$ & $4(8.2)$ & $6(3.9)$ \\
\hline Anticholinergic drug & $0(0.0)$ & $0(0.0)$ & $2(4.1)$ & $2(1.3)$ \\
\hline COMT inhibitor & $5(9.1)$ & $2(4.2)$ & $1(2.0)$ & $8(5.3)$ \\
\hline Antidementia drug & $42(76.4)$ & $36(75.0)$ & $36(73.5)$ & $114(75.0)$ \\
\hline Yokukansan $^{a}$ & $11(20.0)$ & $11(22.9)$ & $13(26.5)$ & $35(23.0)$ \\
\hline Other CNS drug & $20(36.4)$ & $23(47.9)$ & $14(28.6)$ & $57(37.5)$ \\
\hline \multicolumn{5}{|l|}{ Total scores at baseline } \\
\hline UPDRS part 1 & $5.2 \pm 2.7$ & $5.2 \pm 2.8$ & $5.0 \pm 2.3$ & $5.1 \pm 2.6$ \\
\hline UPDRS part 2 ("on") & $12.7 \pm 6.3$ & $13.3 \pm 7.6$ & $13.9 \pm 6.6$ & $13.3 \pm 6.8$ \\
\hline UPDRS part 3 & $31.4 \pm 10.3$ & $33.2 \pm 13.4$ & $32.4 \pm 10.5$ & $32.3 \pm 11.4$ \\
\hline UPDRS part 4 & $1.5 \pm 1.8$ & $1.8 \pm 2.2$ & $1.5 \pm 1.5$ & $1.6 \pm 1.8$ \\
\hline UPDRS parts 1-4 & $50.8 \pm 16.5$ & $53.5 \pm 22.6$ & $52.7 \pm 16.6$ & $52.3 \pm 18.6$ \\
\hline
\end{tabular}


Table 1 Patient characteristics (modified intention-to-treat population) (continued)

\begin{tabular}{|c|c|c|c|c|}
\hline & \multirow[b]{2}{*}{ Placebo $(n=55)$} & \multicolumn{2}{|c|}{ Zonisamide } & \multirow[b]{2}{*}{ Total $(n=152)$} \\
\hline & & $25 \mathrm{mg}(\mathrm{n}=48)$ & $50 \mathrm{mg}(\mathrm{n}=49)$ & \\
\hline MMSE & $21.5 \pm 4.7$ & $21.4 \pm 5.8$ & $21.2 \pm 3.8$ & $21.4 \pm 4.8$ \\
\hline NPI-10 & $7.3 \pm 8.4$ & $6.3 \pm 8.6$ & $7.7 \pm 8.1$ & $7.1 \pm 8.3$ \\
\hline ZBI & $24.5 \pm 19.6$ & $21.6 \pm 16.4$ & $23.3 \pm 15.0$ & $23.2 \pm 17.2$ \\
\hline
\end{tabular}

Abbreviations: COMT = catechol-O-methyltransferase; DLB = dementia with Lewy bodies; LEDD = levodopa equivalent daily dose; MAO-B = monoamine oxidase B; MMSE = Mini-Mental State Examination; NPI-10 = Neuropsychiatric Inventory-10; UPDRS = Unified Parkinson's Disease Rating Scale; ZBI = Zarit Burden Interview.

Data are shown as $\mathrm{n}(\%)$ or mean \pm SD.

a Traditional Japanese herbal medicine.

zonisamide than placebo. The incidences of AEs related to neurologic and psychiatric disorders such as hallucinations and visual hallucinations, which are common symptoms of DLB, were comparable between zonisamide and placebo (table e-6, links.lww.com/WNL/A180). No clinically significant changes in laboratory test values, vital signs, body weight, or ECG findings were observed in any group.

\section{Classification of evidence}

This study provides Class I evidence that zonisamide (adjunctive to levodopa) improves parkinsonism and is well-tolerated in patients with DLB according to the classification scheme requirements for therapeutic questions. ${ }^{20}$

\section{Discussion}

Zonisamide is effective for the treatment of motor symptoms and wearing-off related to PD. ${ }^{3-6}$ In this study, we found that the UPDRS part 3 total score at week 12 was significantly improved in the zonisamide $50 \mathrm{mg} / \mathrm{d}$ group compared with the placebo group of patients with DLB. The effect size of

Table 2 Adverse events (AEs) (safety population)

\begin{tabular}{|c|c|c|c|c|}
\hline \multirow[b]{2}{*}{ Number of patients } & \multirow[b]{2}{*}{ Placebo $(n=58)$} & \multicolumn{3}{|c|}{ Zonisamide } \\
\hline & & $25 \mathrm{mg}(\mathrm{n}=51)$ & $50 \mathrm{mg}(\mathrm{n}=49)$ & Total $^{\mathrm{a}}(\mathrm{n}=100)$ \\
\hline \multicolumn{5}{|l|}{ Overall AEs } \\
\hline Any AEs & $29(50.0) ; 42$ & $22(43.1) ; 41$ & $32(65.3) ; 57$ & $54(54.0) ; 98$ \\
\hline AEs leading to death & $0(0.0)$ & $0(0.0)$ & $0(0.0)$ & $0(0.0)$ \\
\hline Serious AEs & $3(5.2) ; 3$ & 4 (7.8); 5 & $3(6.1) ; 4$ & $7(7.0) ; 9$ \\
\hline AEs leading to discontinuation & $2(3.4) ; 2$ & $5(9.8) ; 6$ & $6(12.2) ; 6$ & $11(11.0) ; 12$ \\
\hline \multicolumn{5}{|l|}{ Common AEs ${ }^{b}$} \\
\hline Dental caries & $0(0.0)$ & $2(3.9)$ & $0(0.0)$ & $2(2.0)$ \\
\hline Nasopharyngitis & $4(6.9)$ & $5(9.8)$ & $5(10.2)$ & $10(10.0)$ \\
\hline Contusion & $5(8.6)$ & $3(5.9)$ & $1(2.0)$ & $4(4.0)$ \\
\hline Excoriation & $0(0.0)$ & $0(0.0)$ & $3(6.1)$ & $3(3.0)$ \\
\hline Weight decreased & $0(0.0)$ & $1(2.0)$ & $4(8.2)$ & $5(5.0)$ \\
\hline Appetite decreased & $1(1.7)$ & $2(3.9)$ & $3(6.1)$ & $5(5.0)$ \\
\hline Somnolence & $2(3.4)$ & $1(2.0)$ & $1(2.0)$ & $2(2.0)$ \\
\hline Rash & $0(0.0)$ & $0(0.0)$ & $2(4.1)$ & $2(2.0)$ \\
\hline
\end{tabular}

Values are $\mathrm{n}(\%)$; number of events.

${ }^{\mathrm{a}} \mathrm{AEs}$ in both zonisamide groups combined.

${ }^{b}$ In $\geq 3 \%$ of patients in either group. 
zonisamide $50 \mathrm{mg} / \mathrm{d}$ for DLB parkinsonism observed in this study was similar to that for PD parkinsonism. ${ }^{3-5}$

We also demonstrated that zonisamide $50 \mathrm{mg}$ significantly improved UPDRS part 3 total scores compared with placebo using both ANCOVA of the PP population and MMRM analysis of the mITT population. Therefore, the results of the analysis using the LOCF method appear to be robust.

UPDRS part 3 total scores decreased from week 4 onward in all groups. The scores remained unchanged up to week 12 in the placebo group whereas a further reduction was observed in the zonisamide 25 and $50 \mathrm{mg}$ groups. Therefore, adjunct zonisamide $50 \mathrm{mg}$ to levodopa therapy may improve motor symptoms in patients with DLB from week 8 of treatment.

In this phase 2 study, the incidence and severity of resting tremor at baseline were lower than those of other symptoms such as rigidity and akinesia/bradykinesia (table e-3, links. lww.com/WNL/A180). This result is consistent with those described in a review by McKeith et al., ${ }^{2}$ where the incidence of resting tremor was lower in patients with DLB than in patients with PD.

The changes from baseline in MMSE, NPI-10, and ZBI total scores were not significant in any of the 3 groups, indicating that zonisamide did not worsen cognitive symptoms, BPSD, or the burden of caregivers.

The incidence of AEs was comparable between the zonisamide $25 \mathrm{mg}$ group and the placebo group, but was higher in the zonisamide $50 \mathrm{mg}$ group compared with placebo. The incidences of weight decreased and appetite decreased were higher in the zonisamide 25 and $50 \mathrm{mg}$ groups compared with placebo. These events are known treatment-related AEs of zonisamide in patients with PD; therefore, we observed no previously unreported types of treatment-related AEs in patients with DLB.

There was no AE for which the severity intensified with increased zonisamide dose. The incidence of somnolence, a common $\mathrm{AE}$ in zonisamide-treated patients with $\mathrm{PD}$, was comparable between the zonisamide groups and placebo group in patients with DLB.

Hallucinations and delusions are the most common psychiatric symptoms observed with anti-Parkinson drugs for the treatment of motor symptoms in patients with DLB. The incidence of neurologic and psychiatric AEs, such as cognitive disorder, hallucinations, and delusions, did not differ between the zonisamide groups and the placebo group, which is consistent with the results of the MMSE and NPI-10 evaluations.

Although these findings suggest zonisamide is clinically safe and useful for the treatment of parkinsonism in patients with DLB, it should be noted that this study was performed with a relatively small number of patients. Further studies are needed to investigate the efficacy of zonisamide in a large number of participants.

\section{Author contributions}

Miho Murata: study design, medical advice, writing/revising, scientific review. Toshinari Odawara: study design, medical advice, scientific review. Kazuko Hasegawa: study design, medical advice, scientific review. Sayaka Iiyama: data analysis/ summary, scientific review. Masatoshi Nakamura: statistical analysis/summary, scientific review. Masaaki Tagawa: writing/revising, scientific review. Kenji Kosaka: study design, medical advice, scientific review.

\section{Acknowledgment}

The authors thank the coinvestigators who participated in this study (listed in the appendix); the following members of Sumitomo Dainippon Pharma Co., Ltd.: Yoshimi Takegawa and Tomoe Kondo for forwarding the clinical trial and Noriko Sato, Yumi Gondo, Hidenori Maruyama, and Osamu Konishi for supporting the manuscript preparation; and J. Ludovic Croxford, PhD, of Edanz Medical Writing, and ELMCOM $\otimes /$ Elsevier, for providing medical writing services.

\section{Study funding}

Supported by Sumitomo Dainippon Pharma Co., Ltd.

\section{Disclosure}

M. Murata received honoraria for consulting and/or lecturing from Sumitomo Dainippon Pharma Co., Ltd., Otsuka Pharmaceutical Co., Ltd., Kyowa Hakko Kirin Co., Ltd., Nippon Boehringer Ingelheim Co., Ltd., Nihon Medi-Physics Co., Ltd., FUJIFILM Pharma Co., Ltd., Hisamitsu Pharmaceutical Co., Inc., and AbbVie GK. Dr. Murata received grants from the Ministry of Education, Culture, Sports, Science and Technology of Japan, the Japan Agency for Medical Research and Development, and the Ministry of Health, Labour and Welfare of Japan. T. Odawara received honoraria for consulting and/or lecturing from Sumitomo Dainippon Pharma Co., Ltd., Ono Pharmaceutical Co., Ltd., Eisai Co., Ltd., Novartis Pharma K.K., Daiichi Sankyo Co., Ltd., Janssen Pharmaceutical K.K., MSD K.K., Eli Lilly Japan K.K., Otsuka Pharmaceutical Co., Ltd., Takeda Pharmaceutical Co., Ltd., Yoshitomiyakuhin Corp., Meiji Seika Pharma Co., Ltd., Astellas Pharma Inc., Nihon Medi-Physics Co., Ltd., Kyowa Hakko Kirin Co., Ltd., and Benesse Corp. Dr. Odawara received grants from the Ministry of Education, Culture, Sports, Science and Technology of Japan, and the Ministry of Health, Labour and Welfare of Japan. K. Hasegawa received honoraria for consulting and/or lecturing from Sumitomo Dainippon Pharma Co., Ltd., Otsuka Pharmaceutical Co., Ltd., Kyowa Hakko Kirin Co., Ltd., Nippon Boehringer Ingelheim Co., Ltd., Hisamitsu Pharmaceutical Co., Inc., and AbbVie GK. Dr. Hasegawa received grants from the Ministry of Health, Labour and Welfare of Japan. S. Iiyama is an employee of Sumitomo Dainippon Pharma Co., Ltd. M. Nakamura is an employee of Sumitomo Dainippon Pharma Co., Ltd. M. Tagawa is an employee of Sumitomo Dainippon Pharma 
Co., Ltd. K. Kosaka received honoraria for consulting and/or lecturing from Sumitomo Dainippon Pharma Co., Ltd., Eisai Co., Ltd., Daiichi Sankyo Co., Ltd., and Nihon Medi-Physics Co., Ltd. Go to Neurology.org/N for full disclosures.

Received March 28, 2017. Accepted in final form November 8, 2017.

\section{References}

1. McKeith IG, Mintzer J, Aarsland D, et al. Dementia with Lewy bodies. Lancet Neurol 2004;3:19-28

2. McKeith IG, Dickson DW, Lowe J, et al. Diagnosis and management of dementia with Lewy bodies: third report of the DLB consortium. Neurology 2005;65:1863-1872.

3. Murata M, Hasegawa K, Kanazawa I; the Japan Zonisamide Study Group. Randomized, double-blind study of zonisamide with placebo in advanced Parkinson's disease. Mov Disord 2004;19:S198.

4. Murata M, Hasegawa K, Kanazawa I; The Japan Zonisamide on PD Study Group. Zonisamide improves motor function in Parkinson disease: a randomized, doubleblind study. Neurology 2007;68:45-50.

5. Murata M, Hasegawa K, Kanazawa I, et al. Randomized placebo-controlled trial of zonisamide in patients with Parkinson's disease. Neurol Clin Neurosci 2016;4:10-15.

6. Murata M, Hasegawa K, Kanazawa I, et al. Zonisamide improves wearing-off in Parkinson's disease: a randomized, double-blind study. Mov Disord 2015;30: 1343-1350.

7. Okada M, Kaneko S, Hirano T, et al. Effects of zonisamide on dopaminergic system. Epilepsy Res 1995;22:193-205.

8. Sonsalla PK, Wong LY, Winnik B, Buckley B. The antiepileptic drug zonisamide inhibits MAO-B and attenuates MPTP toxicity in mice: clinical relevance. Exp Neurol 2010;221:329-334.
9. Murata M. Novel therapeutic effects of the anti-convulsant, zonisamide, on Parkinson's disease. Curr Pharm Des 2004;10:687-693.

10. Suzuki S, Kawakami K, Nishimura S, et al. Zonisamide blocks T-type calcium channel in cultured neurons of rat cerebral cortex. Epilepsy Res 1992;12:21-27.

11. Kito M, Maehara M, Watanabe K. Mechanisms of T-type calcium channel blockade by zonisamide. Seizure 1996;5:115-119.

12. Yamamura S, Ohoyama K, Nagase H, Okada M. Zonisamide enhances delta receptorassociated neurotransmitter release in striato-pallidal pathway. Neuropharmacology 2009;57:322-331.

13. Odawara T, Shiozaki K, Togo T, Hirayasu Y. Administration of zonisamide in three cases of dementia with Lewy bodies. Psychiatry Clin Neurosci 2010;64:327-329.

14. Taguchi Y, Takashima S, Tanaka K. Improvement in parkinsonism with zonisamide treatment in a patient with dementia with Lewy bodies. J Neurol Stroke 2014;1: 00017.

15. Sato S, Mizukami K, Asada T. Successful treatment of extrapyramidal and psychotic symptoms with zonisamide in a patient with dementia with Lewy bodies. Prog Neuropsychopharmacol Biol Psychiatry 2010;34:1130-1131

16. Movement Disorder Society Task Force on Rating Scales for Parkinson's Disease. The Unified Parkinson's Disease Rating Scale (UPDRS): status and recommendations. Mov Disord 2003;18:738-750.

17. Folstein MF, Folstein SE, McHugh PR. "Mini-mental state": a practical method for grading the cognitive state of patients for the clinician. J Psychiatr Res 1975;12: 189-198.

18. Cummings JL, Mega M, Gray K, Rosenberg-Thompson S, Carusi DA, Gornbein J. The Neuropsychiatric Inventory: comprehensive assessment of psychopathology in dementia. Neurology 1994;44:2308-2314.

19. Arai Y, Kudo K, Hosokawa T, Washio M, Miura H, Hisamichi S. Reliability and validity of the Japanese version of the Zarit caregiver burden interview. Psychiatry Clin Neurosci 1997;51:281-287.

20. Gross RA, Johnston KC. Levels of evidence: taking Neurology ${ }^{\circledR}$ to the next level. Neurology 2009;72:8-10. 


\title{
Adjunct zonisamide to levodopa for DLB parkinsonism
}

\author{
A randomized double-blind phase 2 study
}

Miho Murata, MD, PhD, Toshinari Odawara, MD, PhD, Kazuko Hasegawa, MD, PhD, Sayaka liyama, Masatoshi Nakamura, Masaaki Tagawa, PhD, and Kenji Kosaka, MD, PhD

Cite as: Neurology ${ }^{\circledR}$ 2018;90:e664-e672. doi:10.1212/WNL.0000000000005010
Correspondence

Dr. Murata

mihom@ncnp.go.jp

\section{Trial registration number}

JapicCTI-122040.

\section{Study question}

Is zonisamide a safe and effective adjunct to levodopa therapy for parkinsonism in patients with dementia with Lewy bodies (DLB)?

\section{Summary answer}

Adjunctive zonisamide mitigates parkinsonism in patients with DLB and is well-tolerated.

\section{What is known and what this article adds}

Levodopa can mitigate parkinsonism in patients with DLB, but it may induce psychiatric symptoms. Zonisamide is a safe and effective treatment for Parkinson disease, and this study provides Class I evidence that it is an appropriate adjunct for levodopa in patients with DLB.

\section{Participants and setting}

This study examined 173 patients at 60 Japanese centers from March 2013 to April 2014. They were diagnosed with probable DLB, had Unified Parkinson's Disease Rating Scale (UPDRS) part 3 total scores $\geq 10$, and had been taking levodopa for $\geq 12$ weeks.

\section{Design, size, and duration}

This double-blind trial block-randomized 158 patients to a placebo group $\left(\mathrm{n}=58 ; \mathrm{n}_{\text {completed }}=53\right)$, a zonisamide $25 \mathrm{mg}$ group $\left(\mathrm{n}=51 ; \mathrm{n}_{\text {completed }}=42\right)$, and a zonisamide $50 \mathrm{mg}$ group $(\mathrm{n}=49$; $n_{\text {completed }}=42$ ). All groups took tablets orally for 12 weeks.

\section{Primary outcomes}

The primary outcome was the from-baseline change in the UPDRS part 3 total score at week 12 .

\section{Main results and the role of chance}

At 12 weeks, all 3 groups had decreased UPDRS part 3 total scores, indicating mitigation of parkinsonism. Relative to the placebo group, the zonisamide $25 \mathrm{mg}$ group was similar (between-group difference -2.3 ; 95\% confidence interval [CI]

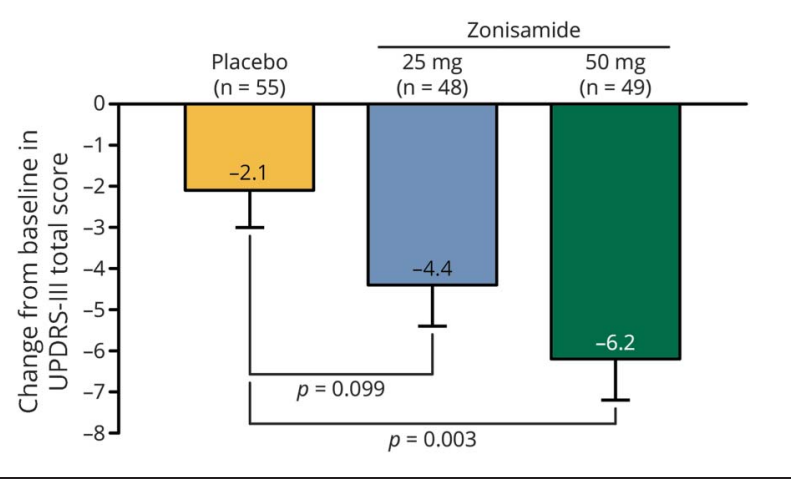

-5.0 to $0.4 ; p=0.099)$, but greater in the zonisamide $50 \mathrm{mg}$ group (between-group difference $-4.1 ; 95 \% \mathrm{CI}-6.8$ to $-1.4 ; p=0.003$ ).

\section{Harms}

The incidence of adverse events in the zonisamide $50 \mathrm{mg}$ group (65.3\%) was higher than those in the zonisamide $25 \mathrm{mg}$ group (43.1\%) and the placebo group (50.0\%). However, zonisamide did not worsen cognitive function or the behavioral or psychological symptoms of dementia.

\section{Bias, confounding, and other reasons for caution}

This study was performed with relatively few patients studied over a short period of time, with minimal ratings for behavioral/psychological symptoms.

\section{Generalizability to other populations}

Between-country differences in genetics, lifestyle, and environmental factors may limit the international generalizability of this study's results.

\section{Study funding/potential competing interests}

This study was funded by Sumitomo Dainippon Pharma, which employs some of the authors. Some authors report receiving honoraria from various pharmaceutical companies, including the study funder, and receiving grants from the Japanese government. Go to Neurology.org/N for full disclosures. 


\section{Neurology}

Adjunct zonisamide to levodopa for DLB parkinsonism: A randomized double-blind phase 2 study

Miho Murata, Toshinari Odawara, Kazuko Hasegawa, et al.

Neurology 2018;90;e664-e672 Published Online before print January 24, 2018

DOI 10.1212/WNL.0000000000005010

This information is current as of January 24, 2018

Neurology ${ }^{\circledR}$ is the official journal of the American Academy of Neurology. Published continuously since 1951, it is now a weekly with 48 issues per year. Copyright $@ 2018$ The Author(s). Published by Wolters Kluwer Health, Inc. on behalf of the American Academy of Neurology.. All rights reserved. Print ISSN: 0028-3878. Online ISSN: 1526-632X.

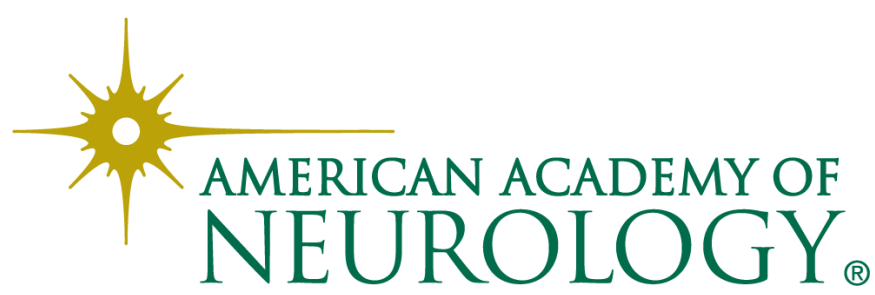




\section{Updated Information \& Services}

References

Citations

Subspecialty Collections

Permissions \& Licensing

\section{Reprints}

including high resolution figures, can be found at: http://n.neurology.org/content/90/8/e664.full

This article cites 20 articles, 4 of which you can access for free at: http://n.neurology.org/content/90/8/e664.full\#ref-list-1

This article has been cited by 1 HighWire-hosted articles: http://n.neurology.org/content/90/8/e664.full\#\#otherarticles

This article, along with others on similar topics, appears in the following collection(s):

All Clinical Neurology

http://n.neurology.org/cgi/collection/all_clinical_neurology Clinical trials Randomized controlled (CONSORT agreement) $\mathrm{http} / / /$ n.neurology.org/cgi/collection/clinical_trials_randomized_contro lled_consort_agreement Dementia with Lewy bodies

http://n.neurology.org/cgi/collection/dementia_with_lewy_bodies Parkinson's disease with dementia http://n.neurology.org/cgi/collection/parkinsons_disease_with_dementi a Parkinson's disease/Parkinsonism http://n.neurology.org/cgi/collection/parkinsons_disease_parkinsonism Information about reproducing this article in parts (figures,tables) or in its entirety can be found online at: http://www.neurology.org/about/about_the_journal\#permissions

Information about ordering reprints can be found online: http://n.neurology.org/subscribers/advertise

Neurology ${ }^{\circledR}$ is the official journal of the American Academy of Neurology. Published continuously since 1951, it is now a weekly with 48 issues per year. Copyright @ 2018 The Author(s). Published by Wolters Kluwer Health, Inc. on behalf of the American Academy of Neurology.. All rights reserved. Print ISSN: 0028-3878. Online ISSN: 1526-632X.

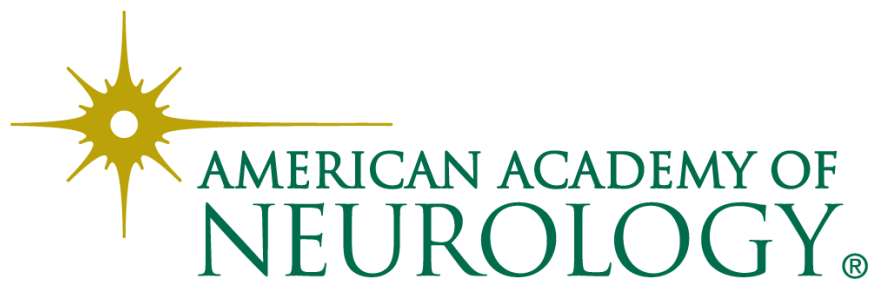

